\title{
Étude de l'apprentissage par la lecture d'étudiants en contexte d'apprentissage par problèmes (APP)
}

\section{SYLVIE CARTIER*}

Université de Montréal

\section{RÉSUMÉ}

Cet article présente les résultats d'une recherche descriptive menée auprès d'étudiants en situation d'apprentissage par la lecture. Il décrit les stratégies rapportées par ceux-ci lorsqu'ils lisent pour acquérir des connaissances reliées à leur domaine de formation. Les résultats indiquent que les stratégies utilisées de façon fréquente par la plupart des sujets sont: (1) les stratégies de lecture telles que la modalité de lire partiellement le texte; (2) les stratégies d'encodage et de rappel telles que la prise de notes et la répétition des informations par la relecture; (3) les stratégies d'autorégulation telles que la planification du travail, l'évaluation de la lecture et du texte ainsi que la contrôle des choix de textes; et (4) les stratégies de gestion des ressources telles que la lecture du livre de référence principal dans le domaine et le choix de la matinée comme moment privilégié d'étude. Or, ces stratégies ne sont pas suffisantes pour supposer un apprentissage significatif tel que le définit le cadre de référence de l'apprentissage par la lecture. Cependant, une analyse des stratégies utilisées à une plus faible fréquence pendant la

\footnotetext{
* Membre du Groupe de recherche interdépartemental sur les conditions d'enseignement et d'apprentissage (GRICEA)
} 
semaine permet de nuancer ces résultats. La conclusion met l'accent sur l'importance de proposer aux étudiants des activités qui leur demandent d'utiliser fréquemment des stratégies efficaces, d'enseigner aux étudiants ces stratégies et de développer le domaine d'étude de l'apprentissage par la lecture.

\section{ABSTRACT}

This article presents the results of a descriptive study of students learning from text. It describes the strategies reported by students during a period of individual work in which they were reading to develop knowledge pertaining to their area of professional training. The results indicate that the strategies used frequently by the majority of subjects were: (1) in reading strategies, the modalities of dealing with the text (partial reading of a chapter); (2) in encoding strategies, re-reading the text in order to retain the information and note taking; (3) in selfregulated strategies, the planning of the work, the evaluation of the reading and the text and the management of changing from one text to another; and (4) in resources management strategies, reading in the principal textbook of the domain and working in the morning. These most frequently used strategies are not sufficient to have effective learning as identify in the conceptual framework on learning from text. However, a more detailed analysis of the strategies used with less frequency during the week reveals nuances in these results. The conclusions stress the importance of selecting activities which require students to use effective strategies more frequently, of teaching them such strategies, and of further developing the field of study of learning from text.

\section{INTRODUCTION}

En ce debut du $21^{\mathrm{e}}$ siècle, le monde du travail se retrouve en profonde mutation: la spécialisation dans les emplois crée des attentes élevées envers les professionnels qui doivent remplir des fonctions variées et spécialisées, ce qui les amène à devoir investir dans leur perfectionnement. Par surcroît, le développement rapide d'informations dans les différents domaines de travail et l'accessibilité croissante de ces 
informations par l'utilisation accrue des nouvelles technologies de l'information et de la communication obligent les professionnels à fréquemment mettre à jour leurs connaissances. Pour se perfectionner dans le cadre de leur formation continue, les professionnels doivent lire de nombreux documents et rapports, des informations sur site Internet ou sur disque compact. Ils sont placés dans des situations que nous appelons l'apprentissage par la lecture. L'apprentissage par la lecture doit non seulement permettre aux professionnels de remplir adéquatement leurs tâches, mais aussi de rester compétitifs sur le marché de l'emploi.

L'apprentissage par la lecture se définit comme «un processus et une situation d'apprentissage dans lesquels le lecteur/apprenant vise à maîtriser un sujet par la lecture de textes «et ce, en gérant à la fois son environnement de travail et la réalisation de la tâche») (Cartier, 2000b, p. 93). Pour ce faire, les sujets doivent avoir recours à un ensemble de stratégies. Une stratégie fait référence à «un ensemble d'actions observables et non observables (comportements, processus, techniques, tactiques) employées par un individu avec une intention particulière et qui sont ajustées en fonction des différentes variables de la situation» (Cartier, 1997, p. 24). En situation d'apprentissage par la lecture, les stratégies à déployer sont: les stratégies d'encodage et de rappel, les stratégies de lecture, les stratégies d'autorégulation et les stratégies de gestion des ressources.

La compétence à lire pour apprendre', nécessaire à tout professionnel, devrait être acquise avant l'arrivée à l'université, car à ce niveau, la grande contribution de la lecture aux apprentissages y est explicitement reconnue (Sublet, 1993). D'ailleurs, pour Hunter (1984), la lecture est l'élément sur lequel repose la qualité de l'enseignement supérieur. Son exploitation devient particulièrement importante dans des programmes novateurs de formation professionnelle, tels que les programmes de médecine basés sur l'apprentissage par problèmes (Bernier \& Des Marchais, 1994). Ces programmes exigent que les étudiants acquièrent une base de connaissances reliée à leur domaine de formation en lisant des ouvrages de référence. Il est donc opportun de se demander ce que font les étudiants lorsqu'ils sont engagés dans des programmes ou dans des cours qui leur demandent de consacrer la 
majorité de leur temps à apprendre par la lecture: Quelles stratégies utilisent-ils spontanément lorsqu'ils doivent lire pour apprendre? Y a-t-il différents profils stratégiques d'apprentissage par la lecture en contexte d'apprentissage par problèmes?

\section{PROBLÉMATIQUE}

Bien que l'apprentissage par la lecture soit caractéristique de différents programmes et cours universitaires, nous constatons, à l'instar de Hunter (1984) et de Vauras (1991), qu'il existe peu d'informations sur cette situation. Hunter (1984) souligne que ce manque d'informations constitue une grave lacune dans le milieu universitaire car, à ce niveau, le processus d'apprentissage est courtcircuité lorsque les stratégies de lecture, d'encodage et de rappel ne sont pas considérées de façon interreliée.

À ce jour, les quelques écrits disponibles portent sur les capacités des étudiants à lire pour apprendre ou se concentrent seulement sur l'étude de certains aspects de cette situation. Dans les écrits portant sur les capacités des étudiants à apprendre par la lecture, un constat s'impose: un bon nombre d'entre eux ne possède pas cette compétence (Cartier, 1997; Simpson, 1984; Sublet, 1993). Cette lacune observée chez les étudiants met en lumière l'importance d'étudier plus en détail l'apprentissage par la lecture. Cette étude permettrait de mieux comprendre les différences entre les étudiants qui démontrent une compétence à lire pour apprendre et ceux qui ont de la difficulté à apprendre en lisant. De plus, cette étude servirait à proposer des pistes d'intervention aux professeurs qui veulent prévenir les problèmes d'apprentissage par la lecture chez leurs étudiants ou encore les aider à y remédier.

Sur une vingtaine de recherche répertoriées sur l'apprentissage par la lecture à l'ordre universitaire, quatre d'entre elles portent sur les stratégies utilisées par des étudiants en situation d'apprentissage par la lecture. Toutefois, ces recherches ne considèrent que certains aspects de cette situation telle que définie dans la présente étude (Entwisle \& Entwisle, 1991; Lahtinen, Lonka \& Lindlom-Ylänn, 1997; Smith, 1982; Wade, Trathen \& Schraw, 1990). 
Or, l'analyse de ces recherches permet de tirer les conclusions suivantes:

- Leur contexte d'expérimentation n'est pas représentatif de la situation de l'apprentissage par la lecture telle qu'elle est définie dans l'introduction de cet article. Les trois premières recherches se limitent à l'étude de l'apprentissage par la lecture d'un seul ou de deux textes spécifiques (Lahtinen, Lonka \& Lindlom-Ylänn, 1997; Smith, 1982; Wade, Trathen \& Schraw, 1990) et la quatrième recherche (Entwisle \& Entwisle, 1991) a été effectuée avec des étudiants au moment de leur préparation à l'examen de certification par leur corporation professionnelle, ce qui correspond davantage à un contexte de révision que d'apprentissage. Selon la définition présentée dans cet article, l'apprentissage par la lecture requiert qu'un apprentissage de contenu soit effectué et ce, par la lecture d'un ou de plusieurs textes ou parties de textes, selon les besoins.

- Ces recherches ont été effectuées à partir de textes traitant de sujets autres que ceux du domaine de formation des étudiants, elles ne représentent donc pas un contexte naturel d'étude. Par exemple, la deuxième recherche (Wade, Trathen \& Schraw, 1990) décrit les stratégies utilisées par les étudiants lorsqu'ils lisent pour apprendre sur les marées dans un texte informatif; ce sujet n'est pas directement relié à leur domaine de formation.

- Enfin, ces recherches ne décrivent pas l'ensemble des stratégies requises par la situation de l'apprentissage par la lecture. Cette situation implique, en plus des stratégies de lecture, des stratégies d'encodage et de rappel, des stratégies d'autorégulation et de gestion des ressources qui permettent la prise en compte de l'organisation et de la gestion du travail et des ressources qui leur sont nécessaires. Les quatre recherches répertoriées décrivent essentiellement les stratégies de lecture, d'encodage et de rappel. 


\section{CADRE DE RÉFÉRENCE}

Le cadre de l'apprentissage par la lecture sert de référence à la présente étude. Pour une description plus détaillée de ce cadre, voir Cartier (1997; 2000b). Le tableau 1 en présente brièvement les composantes.

Ce cadre de référence, fondé sur les domaines de l'apprentissage de texte, de la compréhension en lecture et de l'autorégulation, comprend trois catégories de composantes interreliées: le contexte, le texte et 1'apprenant. Dans la catégorie du contexte figurent les activités d'apprentissage par la lecture. Ces activités, proposées aux étudiants, dirigent leur attention lorsqu'ils traitent les informations contenues dans un texte et les aident à s'ajuster pendant la lecture afin de satisfaire aux exigences de ces activités (Wittrock, 1991).

Dans la catégorie du texte, deux caractéristiques sont retenues en raison de leur influence sur le travail de l'apprenant: la superstructure $d u$ texte (ou grammaire de texte), qui correspond à la structure générale de présentation des idées contenues dans le texte, par exemple, les superstructures argumentative et informative (Deschênes, 1988; van Dijk \& Kintsch, 1983) et l'organisation du texte, qui prend en compte les caractéristiques physiques et textuelles, par exemple, la longueur du texte et la présentation des informations (Jones, 1988; Pressley \& Afflerbach, 1995; Vauras, 1991).

La catégorie de l'apprenant compte six composantes:

1. la conception de l'apprentissage, qui correspond à la conception que l'étudiant se fait de l'acte d'apprendre (Entwisle \& Entwisle, 1991; Romainville, 1993 dans Parmentier \& Romainville, 1998; Säljö, 1984). Dans notre contexte, les différentes conceptions se rangent sur un continuum allant de celle voulant que l'apprentissage consiste à s'attarder sur des détails jusqu'à celle définissant l'apprentissage comme l'analyse du sens global des informations du texte;

2. les connaissances antérieures, qui constituent la base de connaissances que l'apprenant possède en mémoire. Elles sont activées avant d'entreprendre une lecture afin de traiter les nouvelles informations et à différentes étapes de cette 


\section{Tableau 1}

Les composantes du cadre de référence de la lecture pour apprendre à l'ordre universitaire

\begin{tabular}{|c|c|c|c|}
\hline $\begin{array}{l}\text { Activités d'apprentissage } \\
\text { par la lecture: } \\
\text { Apprentissage par problèmes } \\
\text { Présentation d'un exposé } \\
\text { Réalisation d'une recherche } \\
\text { documentaire } \\
\text { Etc. }\end{array}$ & $\begin{array}{l}\text { Connaissances antérieures } \\
\text { Conceptions de l'apprentissage } \\
\text { Stratégies de gestion des ressources pour gérer } \\
\text { le lieu et le moment de travail, de même } \\
\text { que les ressources matérielles et humaines } \\
\text { Stratégies d'autorégulation pour planifier, } \\
\text { contrôler et évaluer la réalisation de } \\
\text { l'apprentissage et des aspects affectifs } \\
\text { Stratégies d'encodage et de rappel pour } \\
\text { acquérir significativement des } \\
\text { connaissances (sélection, répétition, } \\
\text { élaboration et organisation) } \\
\text { Stratégies de lecture pour ressortir les idées } \\
\text { principales et la manière de lire un texte }\end{array}$ & $\begin{array}{l}\text { Superstructure } \\
\text { Organisation }\end{array}$ & 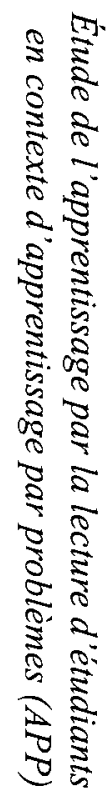 \\
\hline
\end{tabular}


lecture afin d'y intégrer de nouvelles connaissances (Pressley \& Afflerbach, 1995);

3. les stratégies de gestion des ressources, qui constituent différentes façons, pour l'étudiant, de gérer les ressources de son milieu de travail ou d'étude, par exemple, les textes, le moment de travail, les personnes-ressources, etc. (Deschênes, Bourdages, Lebel \& Michaud, 1993; Marcillet, 2000);

4. les stratégies d'autorégulation, qui sont des façons de faire visant la gestion de la réalisation de l'activité d'apprentissage par la lecture et la prise en compte des facteurs affectifs. Ce sont les stratégies de planification, de contrôle et d'autoévaluation (Pressley \& Afflerbach, 1995; Wittrock, 1991; Zimmerman, 1994, 2000);

5 les stratégies d'encodage et de rappel, qui se répartissent selon deux types de traitement en mémoire des informations lues. Elles se situent sur un continuum allant de la reproduction d'informations textuelles selon la structure intégrale du texte (stratégies de répétition) jusqu'à la réalisation d'une version personnalisée ou professionnalisée de la structure des informations (Vauras, 1991). Les stratégies d'organisation (schéma, tableau) et d'élaboration (paraphrase, image, exemple), permettent de faire cette version personnalisée ou professionnalisée des connaissances. Les premières relient les informations du texte entre elles et les structurent en une forme plus facile à encoder pour l'étudiant et les secondes mettent en relation les informations du texte avec les connaissances de l'étudiant. Les stratégies de sélection et de répétition sont d'autres stratégies requises pour réaliser l'encodage et le rappel d'information;

6. les stratégies de lecture, qui sont issues des modèles de la compréhension en lecture, dont ceux de van Dijk \& Kintsch (1983) et de Wittrock (1991). Dans la situation spécifique d'apprentissage par la lecture, l'apprenant doit réduire la densité des informations afin de pouvoir retenir les plus importantes. Pour ce faire, il est important que l'étudiant déploie des stratégies de compréhension qui lui permettent de 
traiter les idées principales contenues dans le texte (ressortir les idées principales, supprimer les idées secondaires, etc.) et qui rendent compte de la manière de lire les textes (complètement, partiellement, etc.).

La présente recherche considère quatre composantes de la catégorie de l'apprenant décrite précédemment dans le cadre de référence: (1) les stratégies de gestion des ressources afin d'identifier efficacement les ressources nécessaires et un contexte de travail adéquat; (2) les stratégies d'autorégulation afin de planifier, de contrôle et d'évaluer la réalisation de l'apprentissage et prendre en compte les aspects affectifs; (3) les stratégies d'encodage et de rappel afin de sélectionner, de répéter, d'élaborer et d'organiser les connaissances et (4) les stratégies de lecture, qui permettent de comprendre les idées principales du texte et qui rendent compte de la façon dont le texte est lu.

\section{OBJECTIFS DE RECHERCHE}

Compte tenu des questions posées dans la problématique et à la lumière du cadre de référence adopté, les objectifs de recherche consistent d'abord à décrire les stratégies qu'utilisent spontanément des étudiants lorsqu'ils lisent pour apprendre lors de périodes de lecture individuelle prévues dans leur programme et, ensuite, à identifier des profils d'apprenant.

\section{MÉTHODOLOGIE}

La présente recherche se situe dans la lignée des recherches en psychopédagogie qui abordent les situations d'apprentissageenseignement dans leur contexte naturel afin d'étudier le fonctionnement cognitif et affectif des apprenants (Wittrock, 1986). Dans ce contexte, le cadre de référence permet d'identifier les stratégies qu'utilisent spontanément des étudiants lorsqu'ils apprennent par la lecture. Pour ce faire, une recherche basée sur l'observation systématique en situation naturelle a été privilégiée (Robert, 1988). 


\section{Contexte de la recherche}

La présente recherche a été menée auprès d'étudiants de troisième année inscrits dans un programme de médecine d'une université québécoise. Dans ce programme, la formule pédagogique privilégiée est l'apprentissage par problèmes. Cette formule exige des étudiants qu'ils acquièrent principalement leurs connaissances par la lecture de textes reliés à l'analyse et à la résolution de problèmes de santé. Les exigences de lecture correspondent à celles de la situation de l'apprentissage par la lecture telle que cet article la définit: les étudiants doivent choisir les textes à lire, gérer leur environnement de travail de même que la réalisation de la tâche et acquérir des connaissances par la lecture de textes.

Pour y arriver, ils doivent analyser le problème étape par étape et émettre des hypothèses de diagnostic au fur et à mesure qu'ils trouvent des indices. Pendant l'analyse du cas, les étudiants doivent lire pour acquérir les connaissances nécessaires à cette activité qui ne sont pas acquises. Les intentions de lecture des étudiants varieront et commanderont différentes façons de lire et d'apprendre. Par exemple, si les étudiants ont l'intention d'éclaircir un détail dans le problème ou de saisir le problème dans son ensemble, les stratégies utilisées différeront. Cette démarche de formation exige aussi des étudiants qu'ils produisent un schéma intégrateur de l'ensemble des connaissances qu'ils ont utilisé incluant leurs nouvelles connaissances.

\section{Échantillon}

Cette recherche a été menée auprès d'un échantillon de quatre étudiantes et deux étudiants. La petite taille de l'échantillon respecte les caractéristiques de la recherche exploratoire et qualitative (Gall, Borg \& Gall, 1996). Elle a été constituée à partir de la technique d'échantillonnage par choix raisonné (Beaud, 1984). Tous les sujets devaient être inscrits à leur cinquième session de formation; avoir été admis au programme la même année; avoir fait leurs études préuniversitaires au Québec; être de langue maternelle française; être sélectionnés dans l'ensemble des groupes qui étudiaient le même problème au même moment et avoir été sélectionnés de façon aléatoire 
parmi l'ensemble des étudiants admissibles à la recherche. Les sujets ont été invités à participer à cette recherche de façon volontaire.

\section{Instruments de recherche}

Les instruments utilisés dans la recherche comprennent le problème à l'étude et les instruments de collecte des données.

\section{Problème à l'étude}

Le problème étudié par les sujets de cette recherche est représentatif des problèmes aigus et chroniques reliés à la consommation d'alcool. Il comprend sept pages de texte décrivant les six premiers jours d'hospitalisation d'un patient. Les quatre premières pages portent sur les notes évolutives de l'hospitalisation du patient, alors que les trois dernières présentent les traitements effectués par les médecins et les résultats obtenus aux tests de laboratoire. Le problème remis aux étudiants comprend aussi un texte de trois pages portant sur les aspects psychologiques associés à la consommation d'alcool. Comme tous les problèmes traités en troisième année de ce programme de médecine, celui-ci est indifférencié, complexe et présente de multiples facettes (Plante, 1995).

\section{Collecte de données}

Deux instruments de collecte de données ont été retenus pour cette recherche, soit le récit de pratique et l'entrevue.

Récit de pratique et données recueillies. Le récit de pratique permet d'étudier le contexte habituel d'apprentissage. Cette procédure, appliquée par d'autres chercheurs tels que Smith (1982), Wade, Trathen \& Schraw (1990) et Lonka, Lindblom-Ylänne \& Maury (1994), est utilisée dans le but d'identifier les activités effectuées et rapportées par les sujets eux-mêmes. Tout comme dans la recherche de Smith (1982), les sujets de cette étude avaient comme principale consigne d'écrire tout ce qu'ils faisaient et ce à quoi ils pensaient pendant leur période d'étude: avant, pendant et après la lecture. En ce sens, des consignes spécifiques 
avaient été remises par écrit aux sujets afin de s'assurer qu'ils couvrent l'ensemble des activités réalisées pendant la semaine.

Entrevue et données recueillies. Une entrevue a été effectuée en vue d'obtenir de l'information sur les stratégies relatives à la lecture pour apprendre. L'entrevue sur les stratégies comprenait deux parties distinctes. La première était effectuée en favorisant la verbalisation du vécu d'action du sujet. Élaborée par Vermersch (1994), ce type de verbalisation permet à la personne d'expliciter une séquence spécifique d'actions telle qu'elle l'a vécue. Ici, les sujets ont eu à décrire la séquence spécifique de leur apprentissage par la lecture. La deuxième partie de l'entrevue servait à préciser des informations reliées aux stratégies, telles que leur efficacité estimée par les sujets, leurs intentions de lecture et d'apprentissage, etc. De plus, les sujets ont été questionnés sur leur façon habituelle de procéder comparativement à celle qu'ils avaient adoptée au cours de la semaine visée par la présente étude. Cette partie de l'entrevue a été effectuée à partir d'un guide d'entrevue préalablement validé. Toutes les entrevues sur les stratégies ont été effectuées par l'expérimentatrice et les entretiens ont été enregistrés sur bande audio.

\section{Déroulement de la recherche}

La recherche auprès des six sujets a eu lieu sur une période de neuf jours. À ce moment, les sujets avaient accumulé deux mois d'expérience d'étude dans la troisième année de médecine. L'expérimentatrice a collecté les données sur une période de neuf jours en suivant les étapes habituelles de travail des sujets. Le tableau 2 présente les trois phases rattachées à la collecte de données servant à décrire les stratégies.

Premièrement, avant la remise du problème choisi, soit le jeudi et le vendredi, l'expérimentatrice a rencontré individuellement les six sujets. Elle leur a présenté par écrit et verbalement les consignes pour réaliser le récit de pratique. Deuxièmement, les étudiants ont eu à rédiger leur récit de pratique, du vendredi au jeudi, pendant leur période d'étude. Ils ont eu à remettre leur récit de pratique le vendredi suivant, à la fin de cette période. Troisièmement, la période d'entrevue sur les stratégies a eu lieu à la fin de la période d'étude, le jeudi (matin et après-midi) ou le 

en contexte d'apprentissage par problèmes (APP)

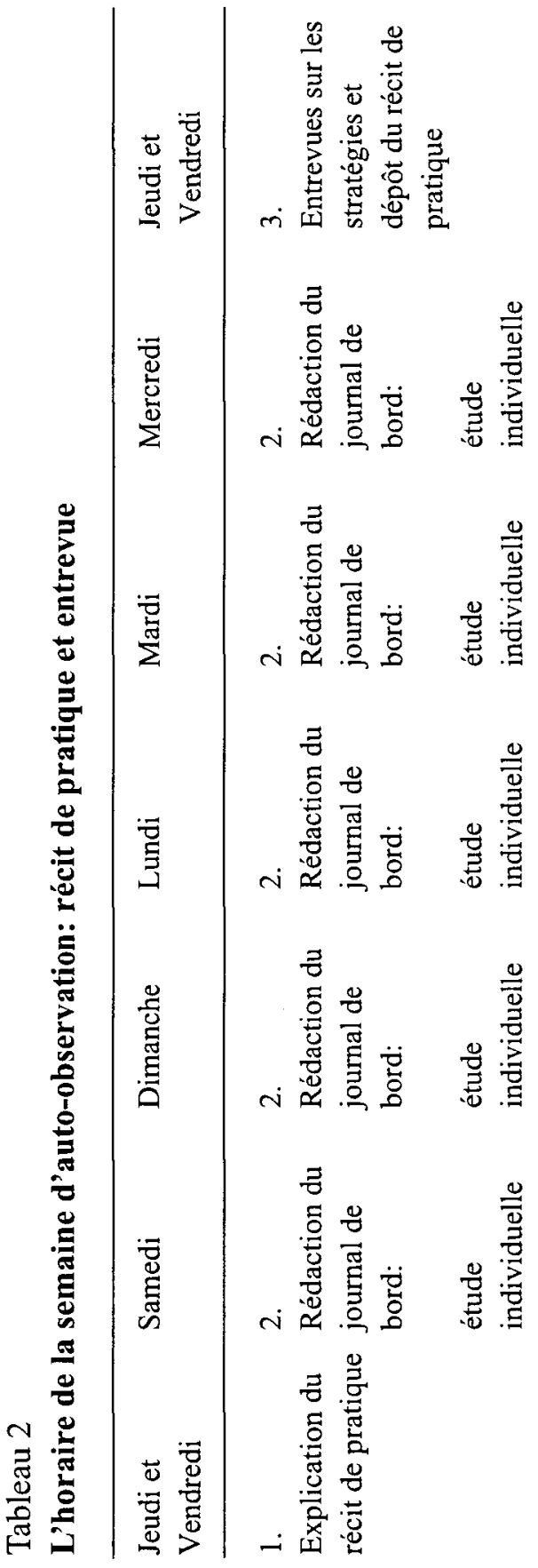


vendredi (matin) afin de contrer les effets possibles reliés au délai. Les entrevues ont duré 75 minutes en moyenne.

\section{Modalités de compilation et d'analyse des résultats}

Dans le but de minimiser le biais voulant que les sujets rapportent moins de stratégies qu'ils n'en utilisent dans l'action (Lonka, LindblomYlänne \& Maury, 1994), la procédure de triangulation des méthodes de collecte de données a été utilisée (Savoie-Zajc, 1993). La description des stratégies rapportées par les sujets dans leur récit de pratique a été complétée par les informations recueillies lors des entrevues.

Le type de compilation des données retenu dans cette recherche est intra-individuelle. Les stratégies ont été compilées et analysées en tenant compte de l'utilisation qu'en faisait individuellement les sujets. Ce procédé permet de voir comment se répartissent les stratégies pour chacun des sujets; il est ainsi possible de déterminer le profil stratégique des sujets.

La démarche de compilation et d'analyse appliquée est quasiqualitative; c'est-à-dire que le contenu qualitatif a été compilé et analysé de façon quantitative (Paillé, 1996). Deux méthodes de compilation des données ont été utilisées, soit le comptage fréquentiel et la mise en évidence de récurrence. Pour effectuer le comptage fréquentiel, la codification des stratégies a d'abord été effectuée à partir d'une grille élaborée à partir de typologies déjà existantes (Cartier, 1997). Seules les stratégies rapportées par les sujets dans la présente recherche ont été conservées dans les tableaux de présentation des résultats. Ensuite, la compilation des stratégies a été faite dans un tableau de fréquence. ${ }^{2}$ Quatre niveaux de fréquence ont servi à l'analyse: très fréquemment ( 75 à 100\%), fréquemment (50 à 74\%), peu fréquemment ( 25 à $49 \%$ ) et rarement ( 0 à $24 \%$ ). Cette compilation des données permet d'avoir accès au profil stratégique de chacun des sujets pour l'ensemble de la période d'apprentissage par la lecture et de vérifier les similitudes et les différences entre chacun. Aussi, ces résultats permettent d'identifier les stratégies utilisées de façon fréquente par les sujets (très fréquemment et fréquemment). 
Pour analyser les récurrences, les stratégies ont été regroupées en neuf catégories d'intentions de lecture observées chez les sujets: par exemple, lire pour poser un diagnostic différentiel, pour faire ressortir les caractéristiques du problème, pour se préparer à l'examen, etc. La compilation de la récurrence des stratégies a été effectuée dans un tableau qui met en évidence deux types d'information: les stratégies utilisées selon deux intentions et plus et les stratégies utilisées selon une seule intention. Cette distinction permet de ressortir les stratégies utilisées de façon discriminative pour une intention de lecture de celles qui sont appliquées de façon plus transversale, pour deux intentions et plus.

Afin de répondre aux objectifs de la présente recherche, deux analyses sont présentées: (1) la description détaillée des stratégies utilisées par les sujets pendant la semaine et (2) l'identification des profils stratégiques des sujets.

\section{RÉSULTATS}

\section{La description détaillée des stratégies}

Les tableaux 3, 4 et 5 présentent les stratégies qui ont été utilisées par les sujets pendant la semaine. Pour chacun des sujets, sont identifiés par des $\mathbf{X}$ majuscules et gras celles qui ont été utilisées de façon fréquente (très fréquemment et fréquemment) ou qui l'ont été selon deux intentions et plus. Lorsque les stratégies n'ont été utilisées que selon une seule intention de lecture, elles sont identifiées par des $\mathrm{v}$ minuscules. Dans la partie qui suit, les principaux résultats sont présentés.

À l'analyse des stratégies de lecture (tableau 3), on peut observer que tous les sujets utilisent de façon fréquente, ou selon deux intentions et plus, différentes modalités de lecture de textes (1), de manières partielle (1.1) et complète (1.2). Quatre sujets ressortent les idées principales du texte (2) en construisant ces idées (2.1) et un seul sujet (C) ressort aussi les idées principales par la suppression d'idées (2.2). Il est toutefois étonnant de constater qu'un sujet (E) ne rapporte jamais utiliser cette stratégie de ressortir les idées principales du texte. De façon générale, les sujets utilisent un éventail assez large de stratégies de lecture et la majorité d'entre eux les utilise de façon fréquente. 


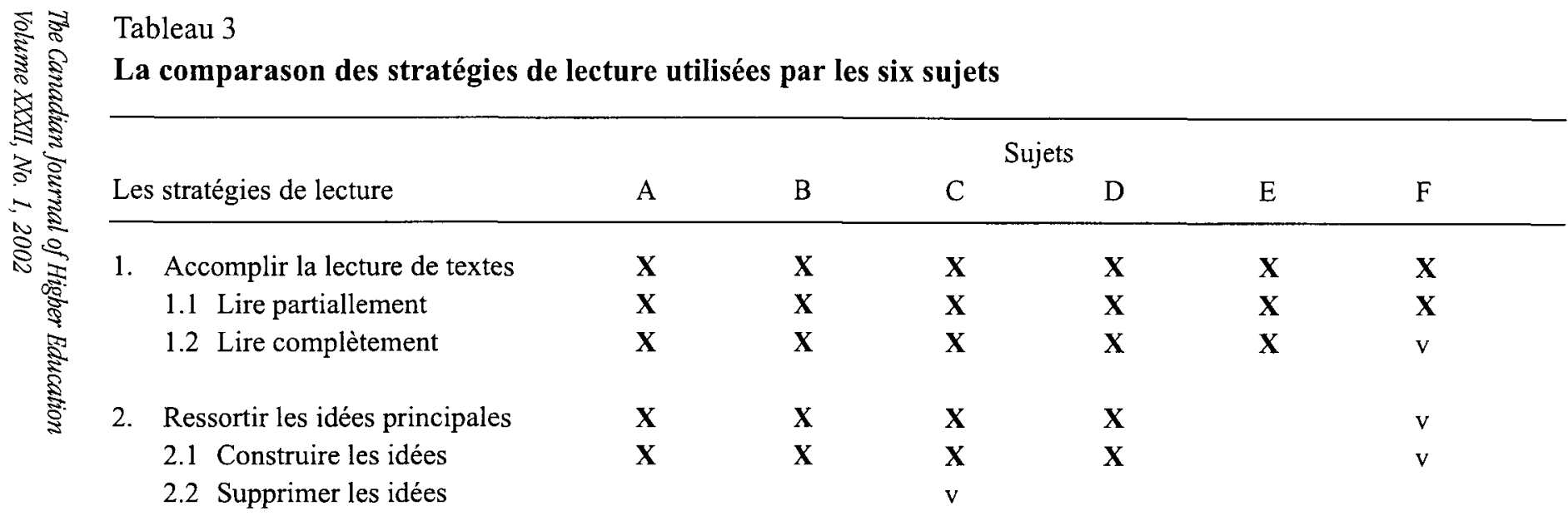

Les $\mathbf{X}$ majuscules et gras indiquent une utilisation fréquente (très fréquemment et fréquemment) ou une utilisation selon deux intentions et plus, alors que les $v$ miniscules signifient que les stratégies ne sont utilisées que selon une seule intention de lecture. 
À l'analyse des stratégies d'encodage et de rappel (tableau 4), on constate que tous les sujets utilisent la répétition (2) de façon fréquente, ou selon deux intentions et plus, notamment par la relecture (2.2) et la catégorie autre (5) dont la prise de notes (5.1). Rappelons que cette stratégie inclut les prises de notes mentionnées par les étudiants et pour lesquelles nous ne connaissions pas le traitement effectué. Est-ce que les notes ont été prises en copiant, en schématisant, sous forme de plan, etc.? Les données recueillies ne permettent pas de clarifier ces informations. Pour ce qui est de la sélection, il est intéressant de noter que toutes les stratégies sont utilisées au moins une fois par l'ensemble des sujets. Par contre, ce sont les stratégies d'organisation (3) et d'élaboration (4) qui semblent les moins utilisées. Plus spécifiquement, en organisation, on observe que les sujets $\mathrm{A}$ et $\mathrm{C}$ ne mentionnent pas utiliser la notation sous forme de schéma (3.2) et que les autres ne le font que selon une seule intention. Il est étonnant que les stratégies d'organisation et d'élaboration soient aussi peu rapportées alors qu'elles sont explicitement encouragées par le programme de formation. Rappelons que les étudiants doivent produire un schéma intégrateur des connaissances reliées au problème à la fin de la semaine et il leur est explicitement demandé d'analyser le problème en se fondant d'abord sur leurs connaissances acquises antérieurement pour ensuite y intégrer les nouvelles connaissances acquises par la lecture.

À l'analyse des stratégies d'autorégulation (tableau 5), il ressort de façon fréquente, ou selon deux intentions et plus, que tous les sujets planifient le travail à effectuer (1.3), évaluent leur lecture (2.2) et les textes (2.3) et contrôlent leur choix de textes. Presque tous les sujets contrôlent aussi de façon fréquente, ou selon deux intentions et plus, leur fonctionnement; sauf le sujet $E$ qui ne le fait que selon une seule intention. On peut aussi observer que quatre sujets (A, B, E, F) planifient l'utilisation de stratégies d'apprentissage (1.1). Ceci porte à penser qu'ils distinguent les moments de lecture et d'apprentissage. Il est étonnant de constater que seulement deux sujets évaluent les apprentissages qu'ils effectuent au courant de la semaine (2.1), alors que cette période d'étude vise justement à acquérir des connaissances. 
La comparason des stratégies d'encodage et de rappel utilisées par les six sujets

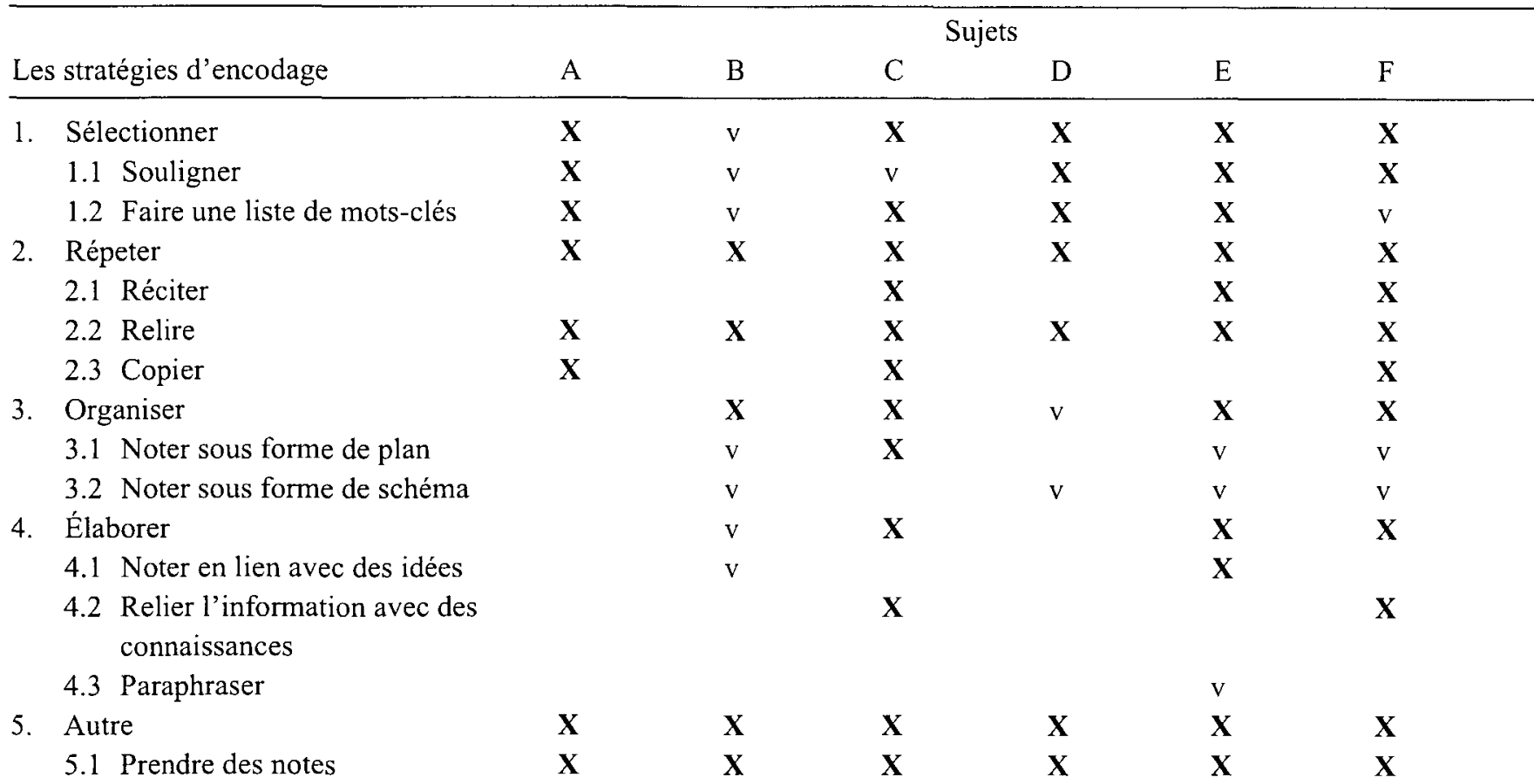

Les $\mathbf{X}$ majuscules et gras indiquent une utilisation fréquente (très fréquemment et fréquemment) ou une utilisation selon deux intentions et plus, alors que les v miniscules signifient que les stratégies ne sont utilisées que selon une seule intention de lecture. 
Etude de l'apprentissage par la lecture d'étudiants

19 en contexte d'apprentissage par problèmes (APP)

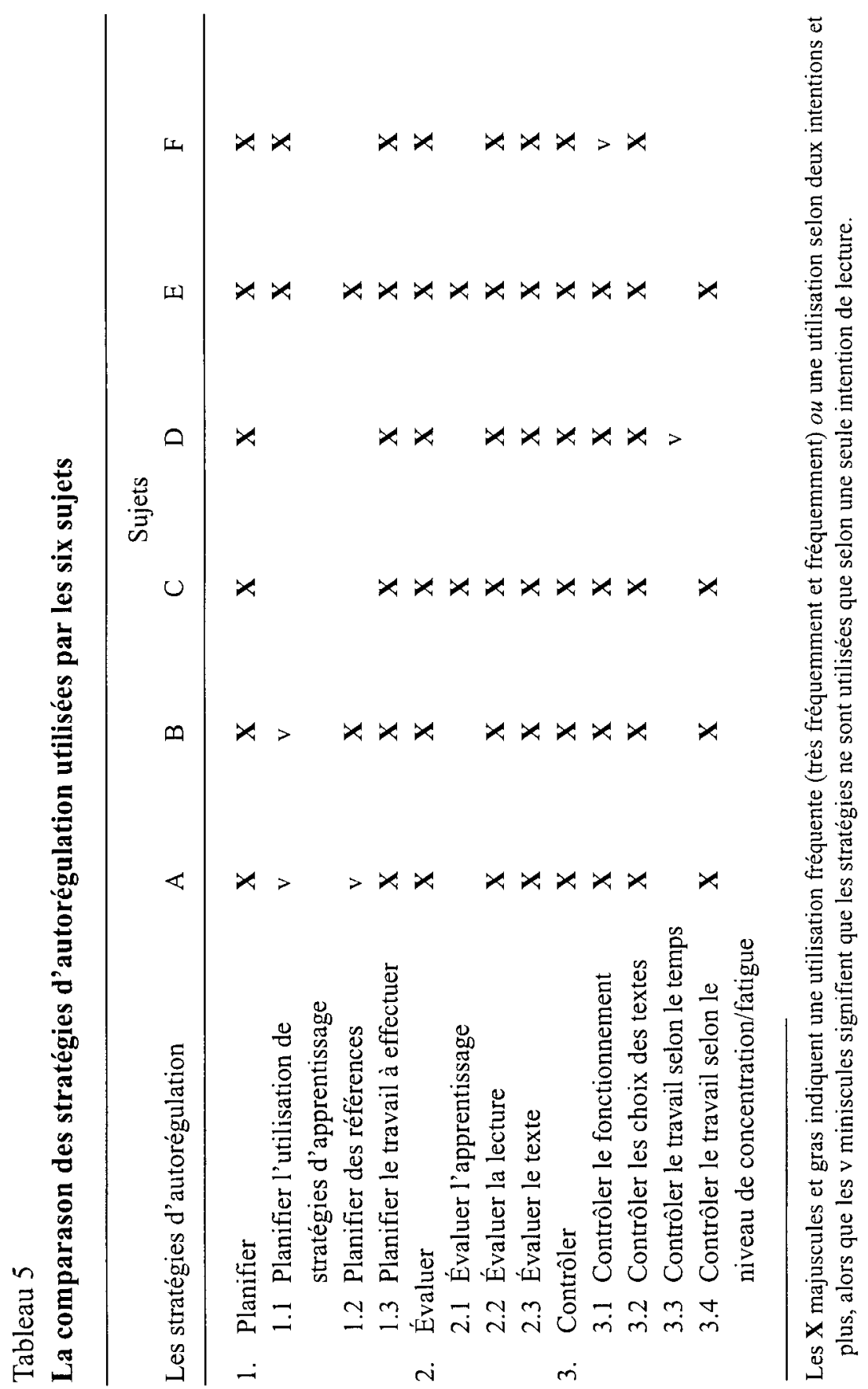

The Canadian Journal of Higher Education Volume XXXII, No. 1, 2002 
Pour l'analyse des stratégies de gestion des ressources, une compilation différente des données a été effectuée. Dans le cas présent, les stratégies qui sont choisies de façon fréquente sont identifiées par des $\mathbf{X}$ majuscules et gras alors que les stratégies utilisées peu fréquemment sont représentées par des $\mathrm{v}$ minuscules. Le tableau 6 présente les stratégies de gestion des ressources utilisées par les six sujets.

À l'analyse des stratégies de gestion des ressources, on peut noter que tous les sujets ont consulté la référence principale en médecine, soit le volume «Harrison», mais qu'un seul sujet (C) l'a fait de façon fréquente. De plus, l'endroit le plus choisi pour travailler par les sujets est le domicile privé, suivi, pour trois d'entre eux, du domicile de leurs parents $(\mathrm{B}, \mathrm{E}, \mathrm{F})$ et pour deux, de la bibliothèque $(\mathrm{A}, \mathrm{C})$. Enfin, pour ce qui est des moments de travail, la majorité des sujets ont travaillé le matin (sauf $A$ ), dont deux de façon peu fréquente (E, F) et un seul sujet a travaillé le soir de façon fréquente (A). Il est intéressant de noter qu'un sujet (D) travaillait la nuit de façon fréquente.

En résumé, six stratégies ont été utilisées de façon fréquente, ou selon deux intentions et plus pendant la semaine, par tous les sujets: la lecture partielle de texte, la répétition par la relecture, la prise de notes, la planification du travail, l'évaluation de la lecture, l'évaluation du texte et le contrôle des choix de textes. De plus, deux stratégies de gestion des ressources ont été utilisées de façon fréquente: la lecture du «Harrison» et l'utilisation du domicile privé comme lieu privilégié de travail.

\section{Identification de profils stratégiques des sujets pour l'ensemble de la période de lecture}

En compilant les résultats par sujet et en les analysant, il est possible d'identifier trois profils stratégiques différents: (1) quatre sujets (A, B, D, E) sélectionnent des textes, lisent, relisent et prennent des notes. Ils organisent et élaborent peu leurs connaissances. De plus, ils contrôlent fréquemment leur choix de textes et leur fonctionnement, car ils trouvent les textes insatisfaisants et ils ont de la difficulté à obtenir les informations nécessaires à l'étude du problème; (2) un sujet (F) cherche principalement à sélectionner des informations dans les textes et à les prendre en note afin de les apprendre à un moment précis (dans le cas 


\section{Tableau 6}

\section{La comparason des stratégies de gestion des ressources utilisées par les six sujets}

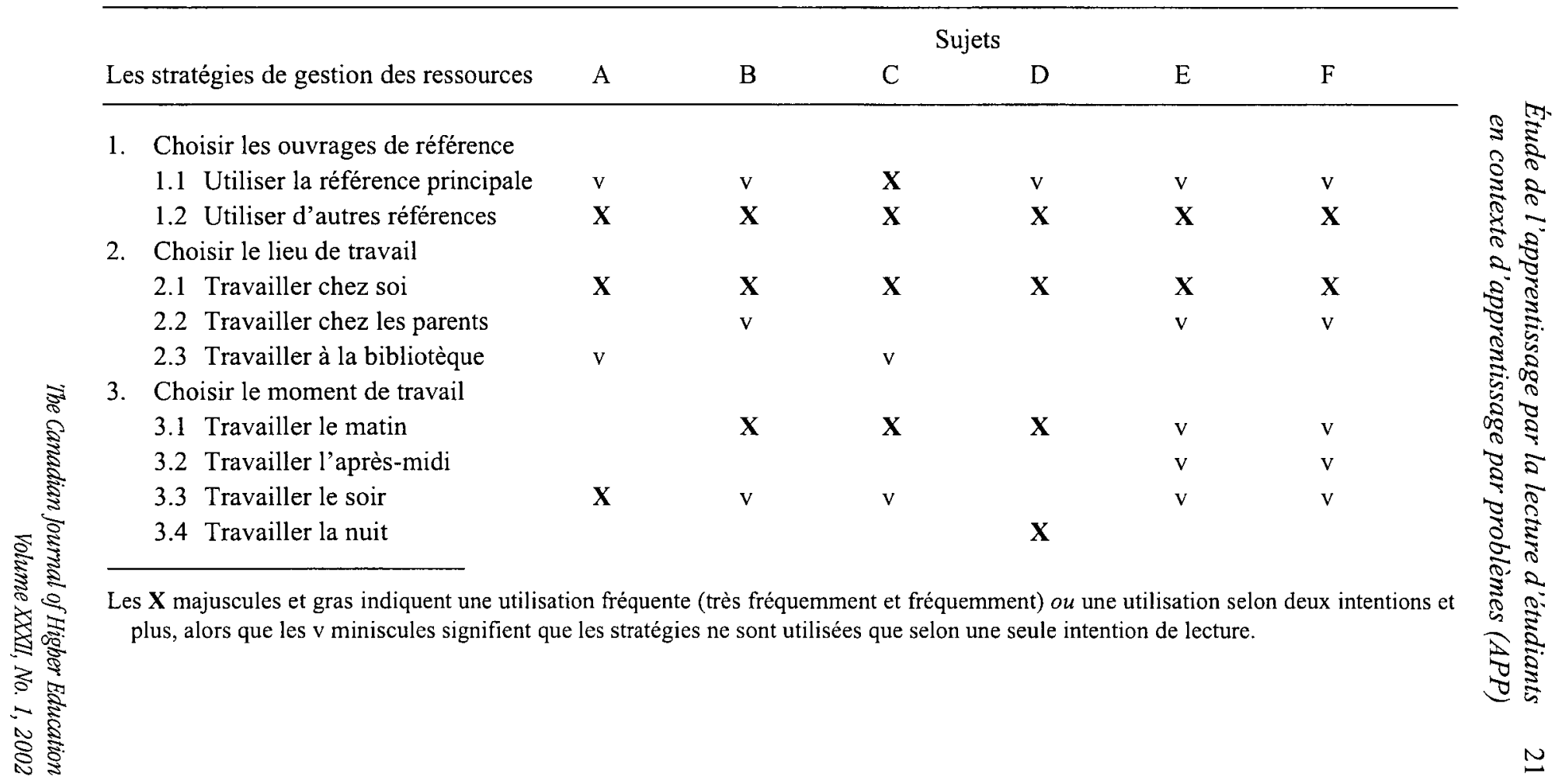


présent, juste avant la rencontre en petit groupe); et (3) un sujet (C) utilise également les stratégies de lecture, d'apprentissage et de métacognition; il lit les informations avec l'intention de trouver d'abord les plus générales et ensuite les plus spécifiques; il utilise toute la gamme des stratégies de lecture et il est celui qui utilise le plus souvent les stratégies qui ressortent les idées principales du texte; pour l'encodage, il est le seul à utiliser de façon fréquente l'ensemble des stratégies qui permettent de sélectionner les idées principales ainsi que de les répéter, les organiser, les élaborer et à prendre des notes; et en métacognition, il est l'un des rares à évaluer ses apprentissages. De plus, il utilise peu la stratégie de planification, seulement pour le travail à effectuer.

L'analyse permet d'observer que ces trois profils de sujets se différencient par le fait que les sujets des deux premiers profils (cinq étudiants sur six) n'utilisent pas de façon fréquente, ou selon deux intentions et plus, l'ensemble des stratégies reconnues efficaces pour construire des connaissances, dont les stratégies d'organisation et d'élaboration. Les sujets du premier profil lisent et relisent pour apprendre alors que le sujet du deuxième profil consigne des informations par écrit pour préparer son étude. Cependant, il est intéressant de noter que l'analyse des stratégies utilisées selon une seule intention fait ressortir que la majorité des sujets utilise l'ensemble des stratégies efficaces; ceci indique qu'ils les connaissent. Quant au troisième profil, il montre un fonctionnement tout à fait adapté à la situation de l'apprentissage par la lecture: il utilise un répertoire diversifié de stratégies en traitant d'abord les idées principales contenues dans les textes, en utilisant des stratégies d'organisation et d'élaboration de même qu'en gérant la réalisation de ses apprentissages.

\section{INTERPRÉTATION}

Les résultats de cette recherche indiquent que les sujets, des étudiants de troisième année de formation en médecine, n'ont pas utilisé de façon fréquente, ou selon deux intentions et plus, l'ensemble des stratégies reconnues efficaces pour apprendre en lisant, bien qu'ils le fassent selon une seule intention. Pourtant, à cette étape de leur 
formation, on aurait pu s'attendre à ce qu'ils aient eu recours spontanément et fréquemment à ces stratégies compte tenu de la complexité du problème à l'étude et des exigences du programme. Quoi qu'il en soit, ce n'est pas ce qu'a révélé l'analyse des données pour cinq étudiants sur six.

Pour interpréter ces résultats, plusieurs hypothèses sont possibles. D'abord, on peut penser que ces cinq sujets ne se sont pas adaptés aux exigences d'intégration de connaissances de cette étape de la formation. Ainsi, ils auraient continué à utiliser les stratégies d'encodage et de rappel apprises et utilisées lors de leurs expériences scolaires antérieures. Ces stratégies devaient être adaptées aux exigences des programmes scolaires qu'ils ont suivis, car ces étudiants en médecine faisaient partie de ceux qui ont eu les moyennes les plus fortes pendant leurs études collégiales au Québec. Lors de la troisième année de formation, les exigences du programme les plaçaient devant des difficultés nouvelles. Dans ce contexte, la plupart n'ont pas utilisé l'ensemble des stratégies requises par les activités et reconnues comme efficaces dans le cadre de référence de l'apprentissage par la lecture. L'utilisation principale des stratégies pour accomplir la lecture de textes ainsi que pour sélectionner et répéter les informations n'est pas suffisante pour intégrer des connaissances. Toutefois, en analysant les stratégies présentes selon une seule intention de lecture, on a pu observer une plus grande diversité de stratégies. Cette observation peut être interprétée de deux façons: ou bien les étudiants commençaient à s'adapter aux particularités de la troisième année du programme de formation (l'intégration de connaissances) ou bien ils effectuaient simplement les tâches demandées, dont la production d'un schéma à la fin de la semaine. Quant au sujet C, qui utilisait l'éventail des stratégies d'apprentissage permettant la construction de connaissances, on peut émettre l'hypothèse qu'il s'était bien adapté aux attentes du programme de formation.

Les exigences de ce programme de médecine, lors de la réalisation de la présente étude, peuvent aussi expliquer ces résultats, entre autres, celles des deux premières années du programme. À ce moment, les problèmes présentés aux étudiants étaient différenciés. Sachant quel système ou organe était visé par le problème, les étudiants pouvaient lire 
sur le sujet sans devoir intégrer les connaissances d'après le contexte clinique, par exemple, sans les relier aux problèmes de santé ou aux symptômes. De plus, pendant ces deux premières années, ils n'avaient pas l'obligation de présenter les informations sous forme de schéma. En troisième année, ils devaient alors changer leur fonctionnement et traiter différemment les problèmes à analyser. Enfin, soulignons que les étudiants de cette étude ont été formés à l'apprentissage par problème dès leur entrée dans le programme. Toutefois, ils n'ont reçu aucune formation sur l'apprentissage par la lecture requise lors de leur étude.

\section{CONCLUSION}

Cet article a présenté les résultats d'une recherche descriptive dont l'objectif consistait à décrire les stratégies utilisées spontanément par six étudiants en médecine en situation d'apprentissage par la lecture. L'expérimentation s'est déroulée dans le contexte naturel de lecture des étudiants, alors qu'ils avaient à lire en vue d'acquérir des connaissances pour analyser et résoudre un problème complexe. La quantité et la qualité des données obtenues par les récits de pratique et les entrevues ont favorisé une analyse en profondeur des résultats. À notre connaissance, aucune recherche n'avait encore été effectuée dans ces conditions. De plus, la compilation des résultats a permis d'identifier trois profils stratégiques d'apprenant, dont deux qui ne réunissent pas l'ensemble des stratégies requises par cette situation d'apprentissage (Cartier, 1997).

Plusieurs constats de cette recherche devraient porter les responsables de programmes de formation basés sur l'apprentissage par la lecture, les professeurs et aussi les chercheurs, à s'interroger. Par exemple, les résultats permettent de penser qu'il ne suffit pas simplement de changer le contexte et les activités d'apprentissage pour que les étudiants changent leur façon d'apprendre. Il faut aussi s'assurer de leur fournir un contexte d'apprentissage qui leur demande vraiment de changer leurs habitudes et leur enseigner à utiliser ces stratégies. Nous recommandons aux responsables des programmes de formation basés sur l'apprentissage par la lecture de s'assurer que leur programme requiert vraiment des 
étudiants qu'ils apprennent en lisant; les étudiants ont des habitudes d'apprentissage qui peuvent être difficiles à changer s'ils ne sont pas convaincus d'avoir à le faire. Depuis 1997, à la suite de cette recherche et d'entrevues effectuées auprès d'étudiants, les responsables du programme ont décidé de changer les exigences des deux premières années de formation. Depuis ce temps, dès leur première année d'étude; les étudiants doivent produire un schéma des informations reliées à chacun des problèmes étudiés. Les responsables souhaitent ainsi que les étudiants travaillent dès le début de leur formation à organiser leurs connaissances en lien avec les problèmes étudiés.

De plus, pour favoriser le changement, nous suggérons d'inclure dans les programmes des activités explicites de formation des étudiants afin de leur indiquer clairement ce que l'on attend d'eux, de leur enseigner les nouvelles stratégies et de leur offrir du «coaching» afin qu'ils expérimentent les apports de ces stratégies à court et à moyen terme. Nous proposons que le «coaching» sur l'apprentissage par la lecture, mené par des professeurs et des étudiants plus expérimentés, formés à l'apprentissage par la lecture, se fasse sous forme d'activités de suivi afin de faire les liens entre les stratégies utilisées et la construction de leurs connaissances. Toujours dans le programme où la recherche a été menée, les étudiants reçoivent maintenant une formation sur l'apprentissage par la lecture à différents moments du programme. De plus, les médecins tuteurs, formés eux aussi à l'apprentissage par la lecture, interviennent directement pour favoriser l'utilisation de cette compétence dans le cadre des activités tutorales. Il deviendra intéressant, dans une perspective de recherche, d'observer ces étudiants en troisième année de formation.

Pour tout professeur ou enseignant préoccupé par l'apprentissage par la lecture de ses étudiants, les résultats de cette recherche sont aussi intéressants, car ils permettent d'obtenir un premier aperçu descriptif de cette situation. Les résultats obtenus peuvent inciter les professeurs et les enseignants à se questionner sur leurs méthodes d'enseignement et sur le rôle qu'ils accordent à la lecture comme situation d'apprentissage. Ils en viendront ainsi à mieux comprendre ce qu'implique cette situation et pourront concevoir des interventions adaptées à la prévention et à la 
correction des difficultés des étudiants. Entre autres, l'utilisation de grilles de lecture peut inciter les étudiants à déployer les stratégies attendues. Mais pour que cela se produise, les questions qui sont posées doivent demander aux étudiants d'organiser les idées principales du texte en lien avec des questions reliées au domaine d'études et de les intégrer à leurs connaissances déjà acquises.

Quant aux chercheurs, ils pourront, grâce au cadre de référence, élargir les domaines de recherche relatifs à la lecture et à l'apprentissage. La situation de l'apprentissage par la lecture ne se limite pas au traitement que fait un apprenant de l'idée d'un auteur. Elle constitue la situation idéale d'acquisition de connaissances, dans laquelle les connaissances sont mises en relation les unes avec les autres. Le développement des connaissances relatives à l'apprentissage par la lecture contribue aussi à approfondir le domaine d'étude de l'autoapprentissage. L'utilisation de la lecture comme situation d'apprentissage est de plus en plus présente en formation continue, et la lecture requise est celle qui demande à l'apprenant d'acquérir des connaissances de façon significative, qu'elles soient théoriques ou pratiques, en lien avec son champ d'expertise professionnelle.

Pour conclure, beaucoup de recherches restent à faire afin de préciser les caractéristiques reliées à l'apprentissage par la lecture selon les domaines d'études, que ce soit dans le cadre de la formation universitaire ou lors de la mise à jour de connaissances. Des recherches devraient être effectuées afin d'expliciter davantage les stratégies utilisées par les étudiants dans cette situation d'apprentissage. Nous espérons que les résultats issus de la présente recherche encourageront les chercheurs à poursuivre l'exploration de ce domaine d'étude.

\section{Les Notes}

1 Dans l'article, les termes «lire pour apprendre», «lire pour construire des connaissances», "apprendre par la lecture» et «la lecture en vue d'apprentissage» sont utilisés indifféremment. 
2 Cette compilation n'implique pas les stratégies de gestion des ressources, car elles sont toujours présentes dans les modalités de travail. Les stratégies de gestion des ressources ne peuvent donc pas se comparer aux autres stratégies qui, par nature, demandent d'être choisies. Le traitement de la fréquence d'utilisation veut justement rendre compte des choix effectués pour le traitement de l'information.

\section{Références}

Beaud, J.-P. (1984). Les techniques d'échantillonnage. Dans B. Gauthier (dir.), Recherches sociales: De la problématique à la collecte des données (pp. 175-200). Sillery, QC: Presses de l'Université du Québec.

Bernier, J.-P., \& Des Marchais, J.E. (1994). Problématiques persistantes d'une nouvelle culture socio-éducative (document inédit). Sherbrooke, QC: Université de Sherbrooke.

Cartier, S. (1997). Lire pour apprendre: description des stratégies utilisées par des étudiants en médecine dans un curriculum d'apprentissage par problèmes. Thèse de doctorat. Montréal, QC: Université de Montréal.

Cartier, S. (2000a). Interventions en classe pour aider les étudiants à mieux apprendre en lisant. Science et comportement, 28(2), 91-104.

Cariter, S. (2000b). La lecture pour apprendre à l'ordre universitaire: Proposition d'un cadre de référence. Res Academica, 18(1 \& 2), 91-104.

Deschênes, A.-J. (1988). La compréhension et la production de texte. Sillery, QC: Les Presses de 1'Université du Québec.

Deschênes, A.-J., Bourdages, C., Lebel, L., \& Michaud, B. (1993). Quelques principes pour concevoir et évaluer des activités d'apprentissage en formation à distance. Revue canadienne de l'éducation, 18(4), 335-348.

Entwisle, N.J., \& Entwisle A. (1991). Contrasting forms of understanding for degree examinations: The student experience and its implications. Higher Education, 22, 205-227.

Gall, M.D., Borg, W.R., \& Gall, J.P. (1996). Educational research: An introduction (6th ed.). New York, NY: Longman.

Hunter, J.S. (1984). Reading: The key to quality in higher education. Reading World, March, 255-264.

Jones, B.F. (1988). Text learning strategy instruction: Guidelines from theory and practice. Dans C.E. Weinstein, E.T. Goetz, \& P.A. Alexander (dir.), Learning and study strategies (pp. 233-260). San Diego, CA: Academic Press. 
Lahtinen, V., Lonka, K., \& Lindblom-Ylänne, S. (1997). Spontaneous study strategies and the quality of knowledge construction. British Journal of Educational Psychology, 67, 13-24.

Lonka, K., Lindblom-Ylänne, S., \& Maury, S. (1994). The effect of study strategies on learning from text. Learning and Instruction, 4(3), 253-271.

Marcillet, F. (2000). Recherche documentaire et information: maitriser l'information. Issy-les-Moulineaux: ESF éditeur.

Paillé, P. (1996). De l'analyse qualitative en général et de l'analyse thématique en particulier. Revue de l'association pour la recherche qualitative, 15, 179-194.

Parmentier, P., \& Romainville, M. (1998). Les manières d'apprendre à l'Université. Dans M. Frenay, B. Noël, P. Parmentier \& M. Romainville (dir.), $L$ 'étudiant apprenant: Grilles de lecture pour l'enseignant universitaire (pp. 63-80). Bruxelles: De Boeck.

Plante, A. (1995). Étude descriptive de l'apprentissage de l'expertise médicale chez des étudiants dans le cadre d'une unité multidisciplinaire en médecine. Mémoire de maîtrise. Sherbrooke, QC: Université de Sherbrooke.

Pressley, M., \& Afflerbach, P. (1995). Verbal protocols of reading: The nature of constructively responsive reading. Hillsdale: Lawrence Erlbaum.

Robert, M. (1988). Fondements et étapes de la recherche scientifique en psychologie (3e éd.). St-Hyacinthe: Edisem.

Säljö, R. (1984). Learning from reading. Dans F. Marton (dir.), The experience of learning (pp. 71-89). Edimbourg: Scottish Academic Press.

Savoie-Zajc, L. (1993). Qu'en est-il de la triangulation: là où la recherche qualitative interprétative se transforme en intervention sociale. Revue de l'Association pour la recherche qualitative, 8, 121-133.

Simpson, M.L. (1984). The status of study strategy instruction: Implications for classroom teachers. Journal of Reading, 28, 136-142.

Smith, S.L. (1982). Learning strategies of mature college learners. Journal of Reading, 26, 5-12.

Sublet, F. (1993). Représentations et pratiques de lecture d'étudiants en lettres et sciences humaines: recherche et proposition pour la formation. Dans E. Fraisse (dir.), Les étudiants et la lecture (pp. 131-152). Paris: PUF.

Van Dijk, T.A., \& Kintsch, W.K. (1983). Strategies of discourse comprehension. London: Academic Press.

Vauras, M. (1991). Text learning strategies in school-aged students. Helsinki: Academia Scientarum Fennica. 
Vermersch, P. (1994). L'entretien d'explicitation: en formation initiale et en formation continue. Paris: ESF.

Wade, S.E., Trathen, W., \& Schraw, G. (1990). An analysis of spontaneous study strategies. Reading Research Quartely, 25(2), 147-166.

Wittrock, M.C. (1986). Preface. Dans M.C. Wittrock (dir.), Handbook of research on teaching (pp. ix-xi). New York, NY: MacMillan.

Wittrock, M.C. (1991). Generative teaching of comprehension. The Elementary School Journal, 92(2), 167-182.

Zimmerman, B.J. (1994). Dimensions of academic self-regulation: a conceptual framework for education. In D.H. Schunk, \& B.J. Zimmerman (dir.), Self-regulation of learning and performance (pp. 3-21). Hillsdale: Lawrence Erlbaum.

Zimmerman, B.J. (2000) Attaining self-regulation: A social perspective. Dans M. Boekaearts, P. Pintrich, \& M. Seider (dir.), Self-regulation theory, research and applications (pp. 13-39). Orlando, FL: Academic Press. 